\title{
Robot-assisted Salvage Pelvic Mass
} Resection

National Cancer Institute

\section{Source}

National Cancer Institute. Robot-assisted Salvage Pelvic Mass Resection. NCI Thesaurus.

Code C161315.

A surgical technique that uses a specially designed robotic system to aid removal of a pelvic mass after failure of initial surgery or other treatment modalities. 